\title{
R\&R- a De novo method to create search terms for IUCr documents
}

Literature search is usually done by a common vocabulary provided by authors, or at times by an ontology to generate information across categories. Web of Science also employs citation indexing to enhance search capabilities such as to search across disciplines. We have developed a new novel Natural Language Procedure that has a potential to automate the identification and indexing of vocabulary for use in a disciplinary community. In this method, vocabulary is created de novo from the documents. The length of the term created by this method could be single terms (nouns) representing a generic concept such as "Material" that would be related to large number of documents or terms that are longer such as "Dielectric material or "high-voltage dielectric material", conjugated compound terms with qualifiers and qualified (root) terms, that are found in a smaller set of documents. Our method uses some of the novel concepts for creating compound words of desired semantics found in Indo-European languages, in particular Sanskrit. The method is called Root and Rule (R\&R, DOI: 10.1007/s11837-015-1487-4). The created terms are specific to the usage in a particular domain and hence providing a means to let the user choose the term her is searching for without needing to develop search strategy as in general purpose search and indexing models. We have been testing our method in many disciplines including Material science, Biology, Cyber security, Engineering and Bibliographic data. One of our test case is the articles held by IUCr where we also use the IUCr common vocabulary as an add on to the R\&R terms. Primary focus of my presentation is to get a community feedback on the vocabulary we created to search IUCr documents.

\section{https://randr.nist.gov/mgi/Default.aspx?dataSource=IUCR}

Talapady N Bhat ${ }^{1 *}$, Jacob Collard ${ }^{1}$, Subrahmanian Eswaran ${ }^{1}$, Ira Monarch ${ }^{1}$, John Elliott ${ }^{1}$, Peter R. Strickland², Brian McMahon ${ }^{2}$

${ }^{1}$ NIST, Gaithersburg, MD 20899, USA

${ }^{2} \mathrm{luCr}$, 5Abbey Square, Chester, CH1 2HU, UK 